\title{
Superconductivity and Superfluidity-Part I: The development of the science of superconductivity and superfluidity in the 20th century
}

\author{
Boris V.Vasiliev \\ *Corresponding Author: bv.vasiliev@narod.ru
}

Copyright (C2013 Horizon Research Publishing All rights reserved.

\begin{abstract}
Currently there is a common belief that the explanation of superconductivity phenomenon lies in understanding the mechanism of the formation of electron pairs. Paired electrons, however, cannot form a superconducting condensate spontaneously. These paired electrons perform disorderly zero-point oscillations and there are no force of attraction in their ensemble. In order to create a unified ensemble of particles, the pairs must order their zero-point fluctuations so that an attraction between the particles appears. As a result of this ordering of zero-point oscillations in the electron gas, superconductivity arises. This model of condensation of zero-point oscillations creates the possibility of being able to obtain estimates for the critical parameters of elementary superconductors, which are in satisfactory agreement with the measured data. On the another hand, the phenomenon of superfluidity in He-4 and He-3 can be similarly explained, due to the ordering of zero-point fluctuations. It is therefore established that both related phenomena are based on the same physical mechanism.
\end{abstract}

Keywords superconductivity superfluidity zero-point oscillations

\section{Introduction}

\subsection{Superconductivity and public}

Superconductivity is a beautiful and unique natural phenomenon that was discovered in the early 20 th century. Its unique nature comes from the fact that superconductivity is the result of quantum laws that act on a macroscopic ensemble of particles as a whole. The concept of superconductivity is attractive not only for circles of scholars, professionals and people interested in physics, but wide educated community.

Extraordinary public interest in this phenomenon was expressed to the scientific community just after the discovery of high temperature superconductors in 1986. Crowds of people in many countries gathered to listen to the news from scientific laboratories. This was the unique event at this time, when the scientific issue was the cause of such interest not only in narrow circle of professionals but also in the wide scientific community.

This interest was then followed by public recognition. One sign of this recognition is through the many awards of Nobel Prize in physics. This is one area of physical science, where plethoras of Nobel Prizes were awarded. The chronology of these awards follows:

1913: Heike Kamerlingh-Onnes was awarded the Nobel Prize in Physics for the discovery of superconductivity;

1962: Lev Landau was awarded the Nobel Prize in Physics in for the pioneering theories for condensed matter, especially liquid helium, or in other words, for the explanation of the phenomenon of superfluidity.

1972: John Bardeen, Leon N. Cooper and J. Robert Schrieffer shared the Nobel Prize in Physics for the development of the theory of superconductivity, usually called the BCS theory.

1973: Brian D. Josephson was awarded the Nobel Prize in Physics in for the theoretical predictions of the properties of the superconducting current flowing through the tunnel barrier, in particular, the phenomena commonly known today under the name of the Josephson effects 
1978: Pyotr Kapitsa was awarded the Nobel Prize in Physics, for his basic inventions and discoveries in the area of low-temperature physics, that is, for the discovery of superfluidity.

1987: Georg Bednorz and Alex Muller received the Nobel Prize in Physics for an important breakthrough in the discovery of superconductivity in ceramic materials.

2003: Alexei Abrikosov, Vitaly Ginzburg and Anthony Legett received the Nobel Prize in Physics for pioneering contributions to the theory of superconductors and superfluids.

Of course, the general attention to superconductivity is caused not just by its unique scientific beauty, but in the hopes for the promise of huge technological advances. These technological advances pave the way, creating improved technological conditions for a wide range of applications of superconductivity in practical societal uses: maglev trains, lossless transmission lines, new accelarators, devices for medical diagnostics and devices based on highly sensitive SQUIDs.

Because of these discoveries, it may now look like there is no need for the development of the fundamental theory of superconductivity at all. It would seem that the most important discoveries in superconductivity have been already made, though more or less randomly.

Isaac Kikoine, a leading Soviet physicist, made a significant contribution to the study of superconductivity on its early on stage. ${ }^{1}$

He used to say, whilst referring to superconductivity, that many great scientific discoveries was made by Columbus method. This was when, figuratively speaking, America was discovered by a researcher who was going to India. This was a way by which Kamerlingh-Onnes came to his discovery of superconductivity, as well as a number of other researchers in this field.

Our current understanding of superconductivity suggests that it is a specific physical discipline. It is the only area of physics where important physical quantities equals exactly to zero. In other areas of physics small and very small values exist, but there are none which are exactly zero. A property can be attributed to the zero value, in the sense there is a complete absence of the considered object. For example, one can speak about a zero neutrino mass, the zero electric charge of neutrons, etc., but these terms have a different meaning.

Is the electrical resistance of superconductors equal to zero?

To test this, H. Kamerlingh-Onnes froze a circulating current in a hollow superconducting cylinder. If the resistance was still there, the magnetic field this current generated would reduced. It was almost a hundred years ago when Kamerlingh Onnes even took this cylinder with the frozen current from Leiden to Cambridge to showcase his findings. No reduction of the field was found.

Now it is clear that resistance of a superconductor should be exactly equal to zero. This follows the fact that the current flow through the superconductor is based on a quantum effect. The behavior of electrons in a superconductor are therefore governed by the same laws as in an atom. Therefore, in this sense, the circulating current over a superconductor ring is analogous to the movement of electrons over their atomic orbits.

Now we know about superconductivity, more specifically it is a quantum phenomenon in a macroscopic manifestation.

It seems that the main obstacles in the way of superconductivity's applications in practice are not the theoretical problems of its in-depth study, but more a purely technological problem. In short, working with liquid helium is too time-consuming and costly and also the technology of nitrogen level superconductors has not yet mastered.

The main problem still lies in correct understanding the physics of superconductivity. Of course, R. Kirchhoff was correct saying that there is nothing more practical than good theory. Therefore, despite the obvious and critical importance of the issues related to the application of superconductors and challenges faced by their technology, they will not be considered.

The most important task of the fundamental theory of superconductivity is to understand the physical mechanisms forming the superconducting state. That is, to find out how the basic parameters of superconductors depend on other physical properties. We also need to overcome a fact that the current theory of superconductivity could not explain why some superconductors have been observed at critical temperature in a critical field.

These were the facts and concepts that have defined our approach to this consideration.

In the first part of it, we focus on the steps made to understand the phenomenon of superconductivity and the problems science has encountered along the way.

The second part of our consideration focuses on the explanation of superconductivity, which has been described as the consequence of ordering of zero-point fluctuations of electrons and that are in satisfactory agreement with the measured data.

${ }^{1}$ I. Kikoine's study of the gyromagnetic effect in the superconductor in the early 1930s led him to determination of the gyromagnetic factor of the superconducting carriers. 


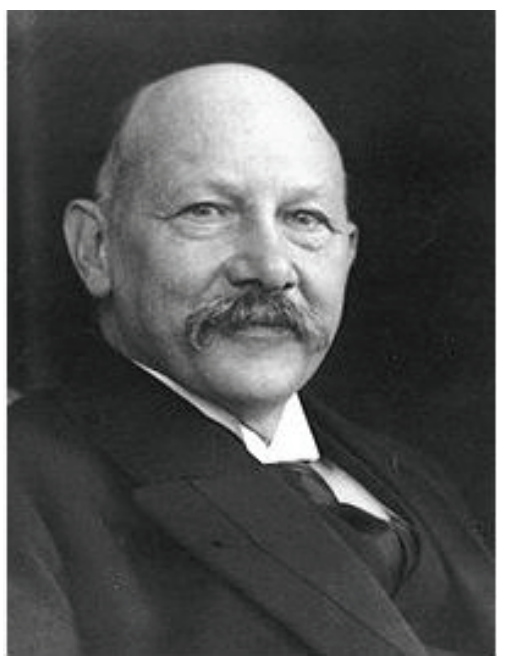

Figure 1. Heike Kamerling-Onnes

The phenomenon of superfluidity in He-4 and He-3 can be similarly explained, due to ordering of zero-point fluctuations.

Thus, it is important that both related phenomena are based on the same physical mechanism.

\subsection{Discovery of superconductivity}

At the beginning of the twentieth century, a new form of scientific research of appeared in the world. Heike Kamerling-Onnes was one of first scientists who used the industry for the service of physics. His research laboratory was based on the present plant of freeze consisting of refrigerators which he developed. This industrial approach gave him complete benefits of the world monopoly in studies at low temperatures for a long time (15 years!). Above all, he was able to carry out his solid-state studies at liquid helium (which boils at atmospheric pressure at $4.18 \mathrm{~K}$ ). He was the first who creates liquid helium in 1908, then he began his systematic studies of the electrical resistance of metals. It was known from earlier experiments that the electrical resistance of metals decreases with decreasing temperature. Moreover, their residual resistance turned out to be smaller if the metal was cleaner. So the idea arose to measure this dependence in pure platinum and gold. But at that time, it was impossible to get these metals sufficiently clean. In those days, only mercury could be obtained at a very high degree of purification by method of repeated distillation. The researchers were lucky. The superconducting transition in mercury occurs at $4.15 \mathrm{~K}$, i.e. slightly below the boiling point of helium. This has created sufficient conditions for the discovery of superconductivity in the first experiment.

One hundred years ago, at the end of November 1911, Heike Kamerlingh Onnes submitted the article [1] where remarkable phenomenon of the complete disappearance of electrical resistance of mercury, which he called superconductivity, was described. Shortly thereafter, thanks to the evacuation of vapor, H. Kamerling-Onnes and his colleagues discovered superconductivity in tin and then in other metals, that were not necessarily very pure. It was therefore shown that the degree of cleanliness has little effect on the superconducting transition.

Their discovery concerned the influence of magnetic fields on superconductors. They therefore determined the existence of the two main criteria of superconductors: the critical temperature and the critical magnetic field. ${ }^{2}$

The physical research at low temperature was started by $\mathrm{H}$. Kamerling-Onnes and has now been developed in many laboratories around the world.

But even a hundred years later, the general style of work in the Leiden cryogenic laboratory created by $\mathrm{H}$. Kamerling-Onnes, including the reasonableness of its scientific policy and the power of technical equipment, still impress specialists.

\section{Basic milestones in the study of superconductivity}

The first twenty two years after the discovery of superconductivity, only the Leiden laboratory of $\mathrm{H}$. KamerlingOnnes engaged in its research. Later helium liquefiers began to appear in other places, and other laboratories were began to study superconductivity. The significant milestone on this way was the discovery of absolute diamagnetism effect of superconductors. Until that time, superconductors were considered as ideal conductors. W. Meissner and

\footnotetext{
${ }^{2}$ Nobel Laureate V.L.Ginzburg gives in his memoirs the excellent description of events related to the discovery of superconductivity. He drew special attention to the ethical dimension associated with this discovery. Ginsburg wrote [2]: "The measurement of the mercury resistance was held Gilles Holst. He was first who observed superconductivity in an explicit form. He was the qualified physicist (later he was the first director of Philips Research Laboratories and professor of Leiden University). But his name in the Kamerling-Onnes's publication is not even mentioned. As indicated in [3], G.Holst itself, apparently, did not consider such an attitude Kamerling-Onnes unfair and unusual. The situation is not clear to me, for our time that is very unusual, perhaps 90 years ago in the scientific community mores were very different."
} 


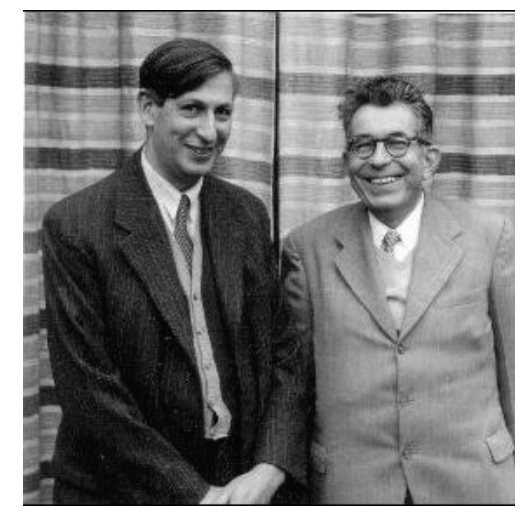

Figure 2. Brothers Heinz and Fritz London

R. Ochsenfeld [4] showed in 1933, that if a superconductor is cooled below the critical temperature in a constant and not very strong magnetic field, then this field is pushed out from the bulk of superconductor. The field is forced out by undamped currents that flow across the surface. ${ }^{3}$

\subsection{The London theory}

The great contribution to the development of the science of superconductors was made by brothers Fritz and Heinz London. They offered its first phenomenological theory. Before the discovery of the absolute diamagnetism of superconductors, it was thought that superconductors are absolute conductors, or in other words, just metals with zero resistance. At a first glance, there is no particulary difference in these definitions. If we consider a perfect conductor in a magnetic field, the current will be induced onto its surface and will extrude the field, i.e. diamagnetism will manifest itself. But if at first we magnetize the sample by placing it in the field, then it will be cooled, diamagnetism should not occur. However, in accordance with the Meissner-Ochsenfeld effect, the result should not depend on the sequence of the events. Inside superconductors the resistance is always:

$$
\rho=0
$$

and the magnetic induction:

$$
B=0 .
$$

In fact, the London theory [5] is the attempt to impose these conditions on Maxwells equations.

The consideration of the London penetration depth is commonly accepted (see for example [7]) in several steps:

Step 1. Firstly, the action of an external electric field on free electrons is considered. In accordance with Newton's law, free electrons gain acceleration in an electric field $\mathbf{E}$ :

$$
\mathbf{a}=\frac{e \mathbf{E}}{m_{e}} .
$$

The directional movement of the "superconducting" electron gas with the density $n_{s}$ creates the current with the density:

$$
\mathbf{j}=e n_{s} \mathbf{v}
$$

where $\mathbf{v}$ is the carriers velocity. After differentiating the time and substituting this in Eq.(3), we obtain the first London equation:

$$
\frac{d}{d t} \mathbf{j}=e n_{s} \mathbf{a}=\frac{n_{s} e^{2}}{m_{e}} \mathbf{E} .
$$

Step 2. After application of operations rot to both sides of this equation and by using Faradays law of electromagnetic induction $\operatorname{rot} \mathbf{E}=-\frac{1}{c} \frac{d \mathbf{B}}{d t}$, we then acquire the relationship between the current density and magnetic field:

$$
\frac{d}{d t}\left(\operatorname{rot} \mathbf{j}+\frac{n_{s} e^{2}}{m_{e} c} \mathbf{B}\right)=0
$$

Step 3. By selecting the stationary solution of Eq.(6)

$$
r o t \mathbf{j}+\frac{n_{s} e^{2}}{m_{e} c} \mathbf{B}=0
$$

\footnotetext{
${ }^{3}$ Interestingly, Kamerling-Onnes was searching for this effect and carried out the similar experiment almost twenty years earlier. The liquefaction of helium was very difficult at that time so he was forced to save on it and used a thin-walled hollow ball of lead in his measurements. It is easy to "freeze" the magnetic field in thin-walled sphere and with that the Meissner effect would be masked.
} 
Table 1. The London penetration depth and the density of superconducting carriers

\begin{tabular}{||c|c|c|c|c||}
\hline \hline $\begin{array}{c}\text { super- } \\
\text { conductors }\end{array}$ & $\begin{array}{c}\lambda_{L}, 10^{-6} \mathrm{~cm} \\
\text { measured } \\
{[8]}\end{array}$ & $\begin{array}{c}n_{s} \\
\text { according to } \\
\text { Eq.(8) }\end{array}$ & $\begin{array}{c}n_{e} \\
\text { in accordance } \\
\text { with Eq.(??) }\end{array}$ & $n_{s} / n_{e}$ \\
\hline $\mathrm{Tl}$ & 9.2 & $3.3 \cdot 10^{21}$ & $1.4 \cdot 10^{23}$ & 0.023 \\
$\mathrm{In}$ & 6.4 & $6.9 \cdot 10^{21}$ & $3.0 \cdot 10^{23}$ & 0.024 \\
$\mathrm{Sn}$ & 5.1 & $1.1 \cdot 10^{22}$ & $3.0 \cdot 10^{23}$ & 0.037 \\
$\mathrm{Hg}$ & 4.2 & $1.6 \cdot 10^{22}$ & $4.5 \cdot 10^{22}$ & 0.035 \\
$\mathrm{~Pb}$ & 3.9 & $1.9 \cdot 10^{22}$ & $1.0 \cdot 10^{24}$ & 0.019 \\
\hline \hline
\end{tabular}

and after some simple transformations, one can conclude that there is a so-called London penetration depth of the magnetic field in a superconductor:

$$
\Lambda_{L}=\sqrt{\frac{m_{e} c^{2}}{4 \pi e^{2} n_{s}}} .
$$

\subsection{The London penetration depth and the density of superconducting carriers}

One of the measurable characteristics of superconductors is the London penetration depth, and for many of these superconductors it usually equals to a few hundred Angstroms [8]. In the Table (1) the measured values of $\lambda_{L}$ are given in the second column.

If we are to use this experimental data to calculate the density of superconducting carriers $n_{s}$ in accordance with the Eq.(8), the results would about two orders of magnitude larger (see the middle column of Tab.(1).

Only a small fraction of these free electrons can combine into the pairs. This is only applicable to the electrons that energies lie within the thin strip of the energy spectrum near $\mathcal{E}_{F}$. We can therefore expect that the concentration of superconducting carriers among all free electrons of the metal should be at the level $\frac{n_{s}}{n_{e}} \approx 10^{-5}$ (see Part-II Eq.(43)). These concentrations, if calculated from Eq.(8), are seen to be about two orders of magnitude higher (see last column of the Table (1)). Apparently, the reason for this discrepancy is because of the use of a nonequivalent transformation. At the first stage in Eq.(3), the straight-line acceleration in a static electric field is considered. If this moves, there will be no current circulation. Therefore, the application of the operation rot in Eq.(6) in this case is not correct. It does not lead to Eq.(7):

$$
\frac{\operatorname{rot} \mathbf{j}}{\frac{n_{s} e^{2}}{m_{e} c} \mathbf{B}}=-1
$$

but instead, leads to a pair of equations:

$$
\begin{aligned}
& \text { rot } \mathbf{j}=0 \\
& \frac{n_{s} e^{2}}{m_{e} c} \mathbf{B}=0
\end{aligned}
$$

and to the uncertainty:

$$
\frac{\operatorname{rot} \mathbf{j}}{\frac{n_{s} e^{2}}{m_{e} c} \mathbf{B}}=\frac{0}{0} .
$$

The correction of the ratio of the Londons depth with the density of superconducting carriers will be given in Part-II Section-7.

\subsection{The Ginsburg-Landau theory}

The London phenomenological theory of superconductivity does not account for the quantum effects.

The theory proposed by V.L. Ginzburg and L.D. Landau [11] in the early 1950s, uses the mathematical formalism of quantum mechanics. Nevertheless, it is a phenomenological theory, since it does not investigate the nature of superconductivity, although it qualitatively and quantitatively describes many aspects of characteristic effects.

To describe the motion of particles in quantum mechanics one uses the wave function $\Psi(r, t)$, which characterizes the position of a particle in space and time. In the GL-theory, such a function is introduced to describe the entire ensemble of particles and is named the parameter of order. Its square determines the concentration of the superconducting particles.

At its core, the GL-theory uses the apparatus, which was developed by Landau, to describe order-disorder transitions (by Landaus classification, it is transitions of the second kind). According to Landau, the transition to 
a more orderly system should be accompanied by a decrease in the amount of free energy:

$$
\Delta W=-a \cdot n_{s}+\frac{b}{2} n_{s}^{2}
$$

where $a$ and $b$ are model parameters. Using the principle of minimum free energy of the system in a steady state, we can find the relation between these parameters:

$$
\frac{d(\Delta W)}{d n_{s}}=-a+b \cdot n_{s}=0 .
$$

Whence

$$
b=\frac{a}{n_{s}}
$$

and the energy gain in the transition to an ordered state:

$$
\Delta W=-\frac{a}{2} n_{s}
$$

The reverse transition from the superconducting state to a normal state occurs at the critical magnetic field strength, $H_{c}$. This is required to create the density of the magnetic energy $\frac{H_{c}^{2}}{8 \pi}$. According to the above description, this equation is therefore obtained:

$$
\frac{H_{c}^{2}}{8 \pi}=\frac{a}{2} n_{s}
$$

In order to express the parameter $a$ of GL-theory in terms of physical characteristics of a sample, the density of "superconducting" carriers generally charge from the London's equation (8). ${ }^{4}$

The important step in the Ginzburg-Landau theory is the changeover of the concentration of superconducting carriers, $n_{s}$, to the order parameter $\Psi$

$$
|\Psi(x)|^{2}=n_{s} .
$$

At this the standard Schrodinger equation (in case of one dimension) takes the form:

$$
-\frac{\hbar}{2 m}[\nabla \Psi(x)]^{2}-a \Psi^{2}(x)+\frac{b}{2} \Psi^{4}(x)=\mathcal{E} .
$$

Again using the condition of minimum energy

$$
\frac{d \mathcal{E}}{d \Psi}=0
$$

after the simple transformations one can obtain the equation that is satisfied by the order parameter of the equilibrium system:

$$
a \Psi+b \Psi|\Psi|^{2}+\frac{1}{4 m_{e}}\left(i \hbar \nabla+\frac{2 e}{c} \mathbf{A}\right)^{2} \Psi=0 .
$$

This equation is called the first Ginzburg-Landau equation. It is nonlinear. Although there is no analytical solution for it, by using the series expansion of parameters, we can find solutions to many of the problems which are associated with changing the order parameter. Such there are consideration of the physics of thin superconducting films, boundaries of superconductor-metal, phenomena near the critical temperature and so on. The variation of the Schrodinger equation (18) with respect the vector potential A gives the second equation of the GL-theory:

$$
\mathbf{j}_{s}=\frac{i \hbar e}{2 m_{e}}\left(\Psi^{*} \nabla \Psi-\Psi \nabla \Psi^{*}\right)-\frac{2 e^{2}}{m_{e} c}|\Psi|^{2} \mathbf{A} .
$$

This determines the density of superconducting current. This equation allows us to obtain a clear picture of the important effect of superconductivity: the magnetic flux quantization.

\section{Experimental data that are important for creation of the theory of superconductivity}

\subsection{Features of the phase transition}

Phase transitions can occur with a jump of the first derivatives of thermodynamic potential and with a jump of second derivatives at the continuous change of the first derivatives. In the terminology of Landau, there are two types of phase transitions: the 1st and the 2nd order. Phenomena with rearrangement of the crystal structure of matter are considered to be a phase transition of the 1st type, while the order-disorder transitions relate to the

\footnotetext{
${ }^{4}$ It should be noted that due to the fact that the London equation does not correctly describes the ratio of the penetration depth with a density of carriers, one should used the revised equation (??) in order to find the $a$.
} 
2nd type. Measurements show that at the superconducting transition there are no changes in the crystal structure or the latent heat release and similar phenomena characteristic of first-order transitions. On the contrary, the specific heat at the point of this transition is discontinuous (see below). These findings clearly indicate that the superconducting transition is associated with a change order. The complete absence of changes of the crystal lattice structure, proven by X-ray measurements, suggests that this transition occurs as an ordering in the electron subsystem.

\subsection{The energy gap and specific heat of a superconductor}

\subsubsection{The energy gap of a superconductor}

Along with the X-ray studies that show no structural changes at the superconducting transition, no changes can be seen in the optical range. When viewing with the naked eye here, nothing happens. However, the reflection of radio waves undergoes a significant change in the transition. Detailed measurements show that there is a sharp boundary in the wavelength range $1 \cdot 10^{11} \div 5 \cdot 10^{11} \mathrm{~Hz}$, which is different for different superconductors. This phenomenon clearly indicates on the existence of a threshold energy, which is needed for the transition of a superconducting carrier to normal state, i.e., there is an energy gap between these two states.

\subsubsection{The specific heat of a superconductor}

The laws of thermodynamics provide possibility for an idea of the nature of the phenomena by means of general reasoning. We show that the simple application of thermodynamic relations leads to the conclusion that the transition of a normal metal-superconductor transition is the transition of second order, i.e., it is due to the ordering of the electronic system.

In order to convert the superconductor into a normal state, we can do this via a critical magnetic field, $H_{c}$. This transition means that the difference between the free energy of a bulk sample (per unit of volume) in normal and superconducting states complements the energy density of the critical magnetic field:

$$
F_{n}-F s=\frac{H_{c}^{2}}{8 \pi} .
$$

By definition, the free energy is the difference of the internal energy, $U$, and thermal energy $T S$ (where $S$ is the entropy of a state):

$$
F=U-T S
$$

Therefore, the increment of free energy is

$$
\delta F=\delta U-T \delta S-S \delta T
$$

According to the first law of thermodynamics, the increment of the density of thermal energy $\delta Q$ is the sum of the work made by a sample on external bodies $\delta R$, and the increment of its internal energy $\delta U$ :

$$
\delta Q=\delta R+\delta F
$$

as a reversible process heat increment of $\delta Q=T \delta S$, then

$$
\delta F=-\delta R-S \delta T
$$

thus the entropy

$$
S=-\left(\frac{\partial F}{\partial T}\right)_{R} .
$$

In accordance with this equation, the difference of entropy in normal and superconducting states (22) can be written as:

$$
S_{s}-S_{n}=\frac{H_{c}}{4 \pi}\left(\frac{\partial H_{c}}{\partial T}\right)_{R} .
$$

Since critical field at any temperature decreases with rising temperature:

$$
\left(\frac{\partial H_{c}}{\partial T}\right)<0
$$

then we can conclude (from equation (28)), that the superconducting state is more ordered and therefore its entropy is lower. Besides this, since at $T=0$, the derivative of the critical field is also zero, then the entropy of the normal and superconducting state, at this point, are equal. Any abrupt changes of the first derivatives of the thermodynamic potential must also be absent, i.e., this transition is a transition of the order-disorder in electron system. 


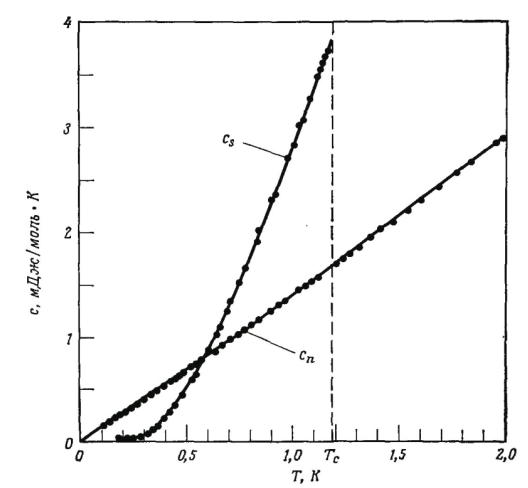

Figure 3. Low-temperature heat capacity of normal and superconducting aluminum[12].

Since, by definition, the specific heat $C=T\left(\frac{\partial S}{\partial T}\right)$, then the difference of specific heats of superconducting and normal states:

$$
C_{s}-C_{n}=\frac{T}{4 \pi}\left[\left(\frac{\partial H_{c}}{\partial T}\right)^{2}+H_{c} \frac{\partial^{2} H_{c}}{\partial T^{2}}\right] .
$$

Since at the critical point $H_{c}=0$, then from (30) this follows directly the Rutgers formula that determines the value of a specific heat jump at the transition point:

$$
C_{s}-C_{n}=\frac{T}{4 \pi}\left(\frac{\partial H_{c}}{\partial T}\right)_{T_{c}}^{2} .
$$

The theory of the specific heat of superconductors is well-confirmed experimentally. For example, the lowtemperature specific heat of aluminum in both the superconducting and normal states supports this in Fig.(3). Only the electrons determine the heat capacity of the normal aluminum at this temperature, and in accordance with the theory of Sommerfeld, it is linear in temperature. The specific heat of a superconductor at a low temperature is exponentially dependent on it. This indicates the existence of a two-tier system in the energy distribution of the superconducting particles. The measurements of the specific heat jump at $T_{c}$ is well described by the Rutgers equation(31).

\subsection{Magnetic flux quantization in superconductors}

The conclusion that magnetic flux in hollow superconducting cylinders should be quantized was firstly expressed F.London. However, the main interest in this problem is not in the phenomenon of quantization, but in the details: what should be the value of the flux quantum. F.London had not taken into account the effect of coupling of superconducting carriers when he computed the quantum of magnetic flux and, therefore, predicted for it twice the amount. The order parameter can be written as:

$$
\Psi(r)=\sqrt{n_{s}} e^{i \theta(r)} .
$$

where $n_{s}$ is density of superconducting carriers, $\theta$ is the order parameter phase.

As in the absence of a magnetic field, the density of particle flux is described by the equation:

$$
n_{s} \mathbf{v}=\frac{i \hbar}{2 m_{e}}\left(\Psi \nabla \Psi^{*}-\Psi^{*} \nabla \Psi\right) .
$$

Using (32), we get $\hbar \nabla \theta=2 m_{e} \mathbf{v}_{\mathbf{s}}$ and can transform the Ginzburg-Landau equation (21) to the form:

$$
\hbar \nabla \theta=2 m_{e} \mathbf{v}_{s}+\frac{2 e}{c} \mathbf{A} .
$$

If we consider the freezing of magnetic flux in a thick superconducting ring with a wall which thickness is much larger than the London penetration depth $\lambda_{L}$, in the depths of the body of the ring current density of $\mathbf{j}$ is zero. This means that the equation (34) reduces to the equation:

$$
\hbar \nabla \theta=\frac{2 e}{c} \mathbf{A} .
$$

One can take the integrals on a path that passes in the interior of the ring, not going close to its surface at any point, on the variables included in this equation:

$$
\hbar \oint \nabla \theta d s=\frac{2 e}{c} \oint \mathbf{A} d s,
$$




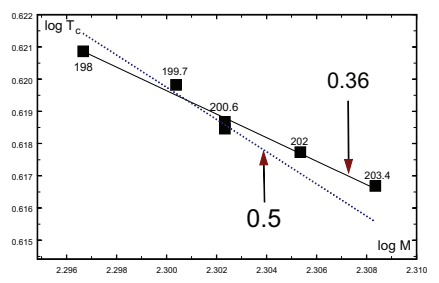

Figure 4. The isotope effect in mercury. The solid line is obtained by the sparse-squares technique. In accordance with the phonon mechanism, the coefficient $\mathfrak{a}$ must be about $1 / 2$ (the dotted line). As it can be seen, this coefficient is in reality approximately equal to $1 / 3$.

and obtain

$$
\oint \nabla \theta d s=\frac{2 e}{\hbar c} \Phi
$$

since by definition, the magnetic flux through any loop:

$$
\Phi=\oint \mathbf{A} d s
$$

The contour integral $\oint \nabla \theta d s$ must be a multiple of $2 \pi$, to ensure the uniqueness of the order parameter in a circuit along the path. Thus, the magnetic flux trapped by superconducting ring should be a multiple to the quantum of magnetic flux:

$$
\Phi_{0}=\frac{2 \pi \hbar c}{2 e}
$$

that is confirmed by corresponding measurements.

This is a very important result for understanding the physics of superconductivity. Thus, the theoretical predictions are confirmed by measurements saying that the superconductivity is due to the fact that its carriers have charge 2e, i.e., they represent two paired electrons. It should be noted that the pairing of electrons is a necessary condition for the existence of superconductivity, but this phenomenon was observed experimentally in the normal state of electron gas metal too. The value of the quantum Eq.(36) correctly describes the periodicity of the magnetic field influence on electron gas in the normal state of some metals (for example, $\mathrm{Mg}$ and $\mathrm{Al}$ at temperatures much higher than their critical temperatures) [9], [10]).

\subsection{The isotope effect}

The most important yet negative role, which plays a major part in the development of the science of superconductivity, is the isotope effect, which was discovered in 1950. The negative role, of the isotope effect is played not just by the effect itself but its wrong interpretation. It was established through an experiment that the critical temperatures of superconductors depend on the isotope mass $\mathrm{Mi}$, from which they are made:

$$
T_{c} \sim \frac{1}{M_{i}^{a}}
$$

This dependence was called the isotope effect. It was found that for type-I superconductors - $\mathrm{Zn}, \mathrm{Sn}, \mathrm{In}, \mathrm{Hg}, \mathrm{Pb}-$ the value of the isotope effect can be described by Eq.(40) at the constant $a=\frac{1}{2}$.

This effect has made researchers think that the phenomenon of superconductivity is actually associated with the vibrations of ions in the lattice. This is because of the similar dependence (Eq.(40)) on the ion mass in order for the maximum energy of phonons to exist whilst propagating in the lattice.

Subsequently this simple picture was broken: the isotope effect was measured for other metals, and it had different values. This difference of the isotope effect in different superconductors could not be explained by phonon mechanism.

It should be noted that the interpretation of the isotope effect in the simple metals did exist though it seemed to fit the results of measurements under the effect of phonons, where $a=1 / 2$. Since the analysis of experimental data [22], [23] (see Fig.(4)) suggests that this parameter for mercury is really closer to $1 / 3$. 


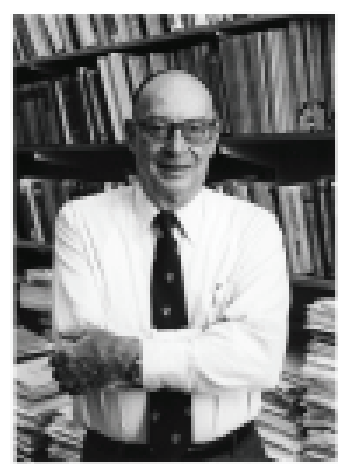

Figure 5. John Bardeen - twice winner of the Nobel Prize. He received the Prize in 1956 for the invention of the transistor, and in 1972, along with L.Cooper and J.Shrieffer, for the creation of the BCS-theory.

\title{
$3.5 \quad \mathrm{BCS}$
}

\begin{abstract}
I would like to put here somewhere my photo at the conversation with John Bardeen. It would be a remarkable illustration of the continuity of generations within the science of superconductivity! The great thing that this photo must exist. In the late 1980s, I was at the conference on superconductivity at Stanford University. I met J. Bardeen, who also attended the conference. It was there that I spoke to him. While we were talking, I saw an American physicist taking a photo of us. I knew this physicist at the time, because he had attend my laboratory in Dubna to acquaint himself with the work of high-TC SQUID. Our laboratory gained international recognition, because we were the first scientific team in the world who could overcome the natural barrier of SQUID sensitivity [18]. This work was aimed at measuring the magnetic cardiogram of humans with the help of high-Tc SQUID. After twenty-odd years now though, I cannot remember neither the name of this American scientist, nor even where he had come from. I hope the photographs taken during his visit to Dubna still exist. So there is still hope that with the help of American friends I can find him, and with him, those photos made more than twenty years ago.

The first attempt to detect the isotope effect in lead was made by the Leiden group in the early 1920s, but due to a smallness of the effect it was not found. It was then registered in 1950 by researchers of the two different laboratories. It created the impression that phonons are responsible for the occurrence of superconductivity since the critical parameters of the superconductor depends on the ion mass. In the same year H.Fröhlich was the first to point out that at low temperatures, the interaction with phonons can lead to nascency of forces of attraction between the electrons, in spite of the Coulomb repulsion. A few years later, L. Cooper predicted the specific mechanism in which an arbitrarily weak attraction between electrons with the Fermi energy would lead to the emergence of a bound state. On this basis, in 1956, Bardeen, Cooper and Shrieffer built a microscopic theory, based on the electron-phonon interaction as the cause of the attractive forces between electrons.
\end{abstract}

It is believed that the BCS-theory has the following main results:

1. The attraction in the electron system arises due to the electron-phonon interaction. As result of this attraction, the ground state of the electron system is separated from the excited electrons by an energetic gap. The existence of energetic gap explains the behavior of the specific heat of superconductors, optical experiments and so on.

2. The depth of penetration (as well as the coherence length) appears to be a natural consequence of the ground state of the BCS-theory. The London equations and the Meissner diamagnetism are obtained naturally.

3. The criterion for the existence of superconductivity and the critical temperature of the transition involves itself the density of electronic states at the Fermi level $\mathcal{D}\left(\mathcal{E}_{F}\right)$ and the potential of the electron-lattice interaction $U$, which can be estimated from the electrical resistance. In the case of $U \mathcal{D}\left(\mathcal{E}_{F}\right) \ll 1$ the BCS-theory expresses the critical temperature of the superconductor in terms of its Debye temperature $\Theta_{D}$ :

$$
T_{c}=1.14 \cdot \Theta_{D} \cdot \exp \left[-\frac{1}{U \mathcal{D}\left(\mathcal{E}_{F}\right)}\right]
$$

4. The participation of the lattice in the electron-electron association determines the effect of isotopic substitution on the critical temperature of the superconductor. At the same time due to the fact that the mass of the isotopes depends on the Debye temperature $\theta_{D} \propto M^{-1 / 2}$, Eq.(41) correctly describes this relationship for a number of superconductors.

5. The temperature dependence of the energy gap of the superconductor is described in the BCS-theory implicitly by an integral over the phonon spectrum from 0 to the Debye energy:

$$
1=\frac{U \mathcal{D}\left(\mathcal{E}_{F}\right)}{2} \int_{0}^{\hbar \omega_{D}} d \xi \frac{t h \sqrt{\xi^{2}+\Delta^{2}} / 2 k T}{\sqrt{\xi^{2}+\Delta^{2}}} .
$$




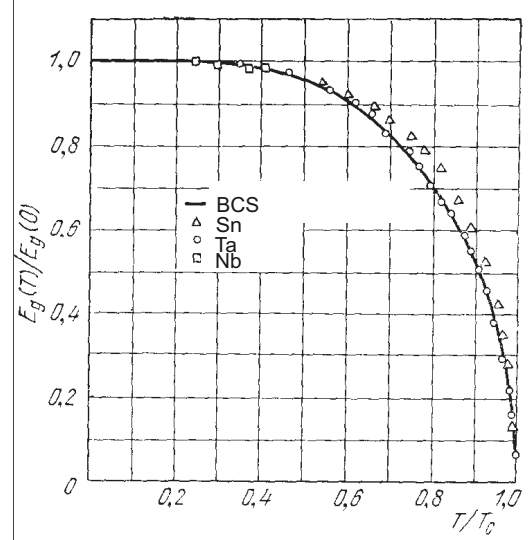

Figure 6. The temperature dependence of the gap in the energy spectrum of superconductors, calculated by the Eq.(42).

The result of calculation of this dependence is in good agreement with measured data(Fig.(6)).

6. The BCS-theory is consistent with the data of measurements of the magnetic flux quantum, as its ground state is made by pairs of single-electron states.

But all this is not in a good agreement with this theory.

First, it does not give the main answer to our questions: with using of it one cannot obtain apriori information about what the critical parameters of a particular superconductor should be. Therefore, BCS cannot help in the search for strategic development of superconductors or in the tactics of their research. Eq.(41) contains two parameters that are difficult to assess: the value of the electron-phonon interaction and the density of electronic levels near the Fermi level. Therefore, BCS cannot be used solely for this purpose, and can only give a qualitative assessment.

In addition, many results of the BCS theory can be obtained by using other, simpler but 'fully correct' methods.

The coupling of electrons in pairs can be the result not only of electron-phonon mechanism. Any attraction between the electrons can lead to their coupling.

For the existence of superconductivity, the bond energy should combine into single ensembles of separate pairs of electrons, which are located at distances of approximately hundreds of interatomic distances. In BCS-theory, there are no forces of attraction between the pairs and, especially, between pairs on these distances.

The quantization of flux is well described within the Ginzburg-Landau theory (see Sec.(3.3)), if the order parameter describes the density of paired electrons.

By using a two-tier system with the approximate parameters, it is easy to describe the temperature dependence of the specific heat of superconductors.

So, the calculation of the temperature dependence of the superconducting energy gap formula (Eq.(42)) can be considered as the success of the BCS theory .

However, it is easier and more convenient to describe this phenomenon as a characterization of the order-disorder transition in a two-tier system of zero-point fluctuations of condensate. In this approach, which is discussed below in Part II, the temperature dependence of the energy gap receives the same interpretation as other phenomena of the same class such as the $\lambda$-transition in liquid helium, the temperature dependence of spontaneous magnetization of ferromagnets and so on.

Therefore, as in the 1950s, the existence of isotope effect is seen to be crucial. However, to date, there is experimental evidence that shows the isotope substitution leads to a change of the parameters of the metals crystal lattice due to the influence of isotope mass on the zero-point oscillations of the ions (see [?]). For this reason, the isotope substitution in the lattice of the metal should lead to a change in the Fermi energy and its impact on all of its electronic properties. In connection with this, the changing of the critical temperature of the superconductor at the isotope substitution can be a direct consequence of changing the Fermi energy without any participation of phonons.

The second part of this book will be devoted to the role of the ordering of zero-point oscillations of electrons in the mechanism of the superconducting state formation. 


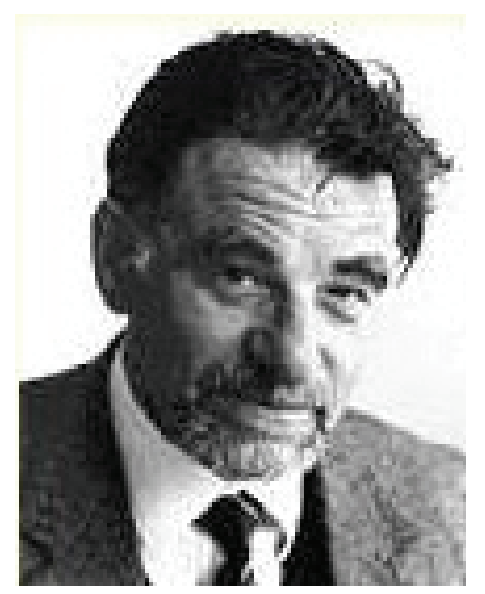

Figure 7. Karl Alexander Muller - founder of HTSC.

Table 2. Critical parameters of superconductors

\begin{tabular}{||c|c|c||}
\hline \hline superconductor & $T_{c}, K$ & $H_{c}, O e$ \\
\hline $\mathrm{Hg}$ & 4.15 & 41 \\
$\mathrm{~Pb}$ & 7.2 & 80 \\
$\mathrm{Nb}$ & 9.25 & 206 \\
$\mathrm{NbTi}$ & $9.5-10.5$ & 120.000 \\
$\mathrm{~N} b_{3} \mathrm{Sn}$ & $18.1-18.5$ & 220.000 \\
$\mathrm{~N} b_{3} \mathrm{Al}$ & 18.9 & 300.000 \\
$\mathrm{~N} b_{3} \mathrm{Ge}$ & 23.2 & 370.000 \\
$\mathrm{MgB}_{2}$ & 40 & 150.000 \\
$\mathrm{YBa}_{2} \mathrm{Cu}_{3} \mathrm{O}_{7}$ & 92.4 & 600.000 \\
$\mathrm{Bi}_{2} \mathrm{Sr}_{2} \mathrm{Ca}_{2} \mathrm{Cu}_{3} \mathrm{O}_{10}$ & 111 & 5.000 .000 \\
$\mathrm{HgBa} \mathrm{Ca}_{2} \mathrm{Cu}_{3} \mathrm{O}_{8}$ & 133 & $>10.000 .000$ \\
\hline \hline
\end{tabular}

\section{The new Era - HTSC}

During the century following the discovery of superconductivity, 40 pure metals were observed. It turned out that among them, Magnesium has the lowest critical temperature of about $0.001 \mathrm{~K}$, and Technetium has the highest at $11.3 \mathrm{~K}$.

Also, it was found that hundreds of compounds and alloys at low temperatures have the property of superconductivity. Among them, the intermetallic compound $\mathrm{Nb}_{3} \mathrm{Ge}$ has the highest critical temperature $23.2 \mathrm{~K}$.

In order to obtain the superconducting state in these compounds it is necessary to use the expensive technology of liquid helium. ${ }^{5}$

Theoretically, it seems that liquid hydrogen could also be used in some cases. But this point is still more theoretical consideration than practical one: hydrogen is a very explosive substance. For decades scientists have nurtured a dream to create a superconductor which would retain its properties at temperatures above the boiling point of liquid nitrogen.

Liquid nitrogen is cheap, accessible, safe, and a subject to a certain culture of working with him (or at least it is not explosive). The creation of such superconductor promised breakthrough in many areas of technology.

In 1986, these materials were found. At first, Swiss researchers A.Muller and G.Bednortz found the superconductivity in the copper-lanthanum ceramics, which temperature of superconducting transition was only 40K, and soon it became clear that it was the new class of superconductors (they was called high-temperature superconductors, or HTSC), and a very large number of laboratories around the world were included in studies of these materials.

One year later, the superconductivity was discovered in ceramic $Y \mathrm{BaCuO}$ with transition temperature higher than $90 \mathrm{~K}$. As the liquid nitrogen boils at $78 \mathrm{~K}$, the nitrogen level was overcome.

Soon after it, mercury based ceramics with transition temperatures of the order of $140 \mathrm{~K}$ were found. The history of increasing the critical temperature of the superconductor is interesting to trace: see the graph (8).

${ }^{5}$ For comparison, we can say that liter of liquid helium costs about a price of bottle of a good brandy, and the heat of vaporization of helium is so small that expensive cryostats are needed for its storage. That makes its using very expensive. 


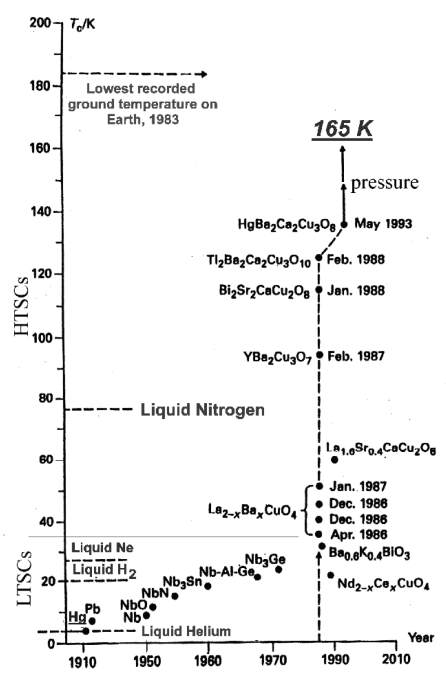

Figure 8. Growth of the critical temperature of known superconductors in time. The horizontal lines indicate the temperature of boiling of cryogenic liquids (at normal pressure).

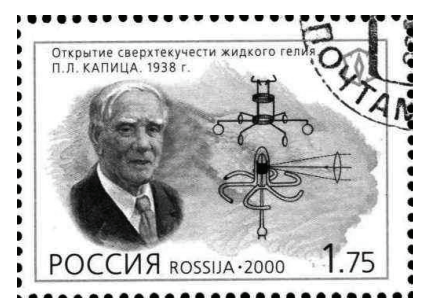

Figure 9. Pyotr L.Kapitsa

It is clear from this graph that if the creation of new superconductors has been continuing at the same rate as before the discovery of HTSC, the nitrogen levels would have been overcome through 150 years. But science is developing by its own laws, and the discovery of HTSC allowed to raise sharply the critical temperature.

However, the creation of high-Tc superconductors has not led to a revolutionary breakthrough in technology and industry.

Ceramics were too low-technological to manufacture a thin superconducting wires. Without wires, the using of high-Tc superconductors had to be limited by low-current instrument technology. It also did not cause the big breakthroughs in this manner, either (see, e.g. [18]).

After discoveries of high-Tc superconductors, no new fundamental breakthrough of this value was made. Perhaps the reason for this is that the BCS-theory, adopted by most researchers to date, can not predict the parameters of superconductors apriori and serves as just a support in strategy and tactics of their research.

\section{About superfuidity}

The first study of the properties of helium-II began in the Leiden laboratory as early as in 1911, the same year when superconductivity was discovered. A little later the singularity in the specific heat, called the $\lambda$-transition, was discovered. However, the discovery of superfluidity of liquid helium was still far away, as Pyotr L. Kapitsa discovered it by 1938 . 


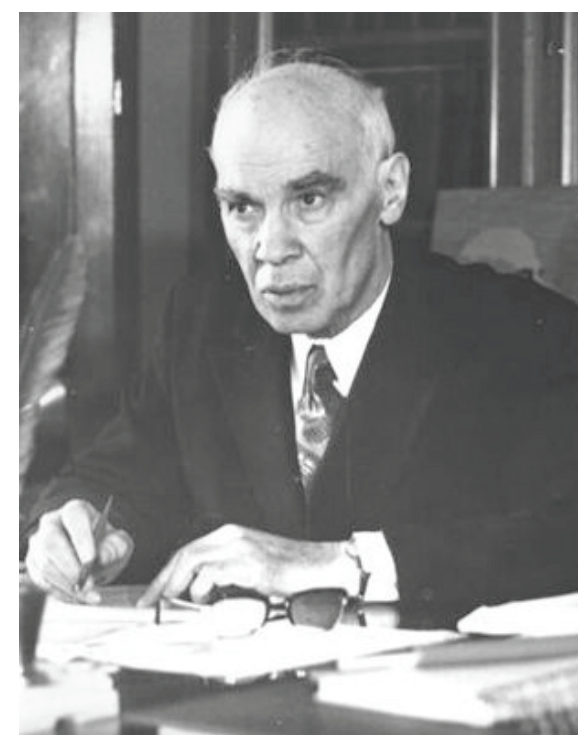

Figure 10. Isaak K.Kikoine

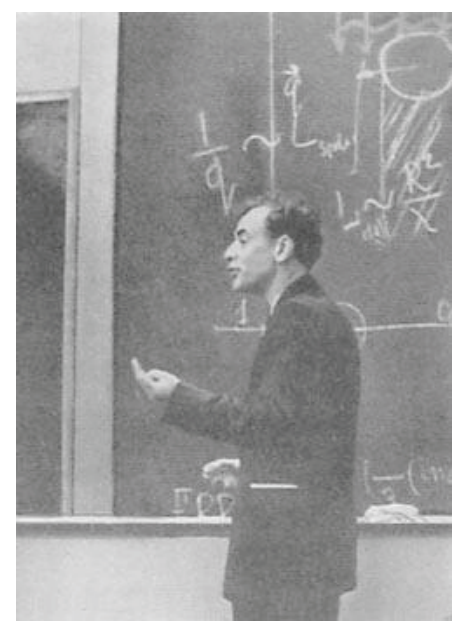

Figure 11. L.D. Landau

\footnotetext{
Here is his story about Kapitsa and superfluidity as it was remembered and relayed to me.
}

It happened in 1933 when Isaac Kikoine was 25 years old. He had just completed his experiment of measuring of the gyromagnetic effect in superconductors. Pyotr Kaptsa was aware of this experiment, he even sent a small ball of super-pure lead for this experiment all the way from the Mond Laboratory in Cambridge, which he led then.

Almost every summer, he went with his family on his own car to Crimea. This was an absolute luxury for Soviet people at that time!

On his way he visited physical laboratories in Moscow and Leningrad (now again St. Petersburg), lecturing and networking with colleagues, friends and admirers. During one of these visits, in 1933, Kikoine had a chance to talk to Kapitsa about results of his measurements. Kapitsa liked these results, and he invited Kikoine to work in Cambridge. They had arranged all formalities, including that Kapitsa will send him an invitation to work in England must be organized for the year. They planned to go to
But it has not happen

Kapitsa's father-in-law was A.N. Krylov was a famous ship-bilt with Stalin himself. Stalin did receive them and asked them

Yesat is the problem?

He will not be released. Becuse the Russian nightingale must sing in Russia!

Pavlov (physiologist) said in reply:

the cage!

Anyway he would sing for us!

So terribly unkind (it is a some understatement yet) Kapitsa became the deputy director of the Leningrad Physical-Technical Institute, which was directed by A.F. Ioffe. Young professors of this institution - Kikoine, Kurchatov, Alikhanov, Artsimovich then went to see the Deputy Director.

Alikhanov asked at the door of the office from outgoing Artsimovich:

Well, how is he? A beast or a man?

This nickname stuck firmly to P. Kapitsa

The scientists of the older generation called him by the same nickname, even decades later.

Stalin, of course, was a criminal. Thanks to his efforts, almost each family in the vast country lost some of its members in the terror of unjustified executions or imprisonments.

However Stalin's role in the history of superfluidity can be considered positive.

All was going well for Kapitsa in Russia,

if to consider researches of superfluidity.

Already after a couple of years, L.Landau ${ }^{6}$ was able to give a theoretical explanation of this phenomenon. He viewed helium-II as a substance where the laws of quantum physics worked on a macroscopic scale. 
This phenomenon is akin to superconductivity: the superconductivity can be regarded as the superfluidity of an electron liquid. As a result, the relationship between phenomena have much in common, since both phenomena are described by the same quantum mechanics laws in macroscopic manifestations. This alliance, which exists in the nature of the phenomena, as a consequence, manifests itself in a set of physical phenomena: the same laws of quantum effects and the same physical description.

For example, even the subtle quantum effect of superconductors, such as the tunneling Josephson effect, manifests itself in the case of superfluidity as well.

However, there are some differences.

At zero temperature, only a small number of all conduction electrons form the superfluid component in superconductors.

The concentration of this component is of the order $\frac{k T_{c}}{\mathcal{E}_{F}} \approx 10^{-5}$, while in liquid helium at $T=0$, all liquid becomes superfluid, i.e., the concentration of the superfluid component is equal to 1.

It is also significant to note that the electrons which have an interaction with their environment have an electric charge and magnetic moment. This is the case yet it seems at first glance that there are no mechanisms of interaction for the formation of the superfluid condensate in liquid helium. Since the helium atom is electrically neutral, it has neither spin, nor magnetic moment.

By studying the properties of helium-II, it seems that all main aspects of the superfluidity has been considered. These include: the calculations of density of the superfluid component and its temperature dependence, the critical velocity of superfluid environment and its sound, the behavior of superfluid liquid near a solid wall and near the critical temperature and so on. These issues, as well as some others, are considered significantly in many high-quality original papers and reviews [14] - [17]. There is no need to rewrite their content here.

However, the electromagnetic mechanism of transition to the superfluid state in helium-4 still remains unclear, as it takes place at a temperature of about one Kelvin and also in the case of helium-3, at the temperature of about a thousand times smaller. It is obvious that this mechanism should be electromagnetic.

This is evidenced by the scale of the energy at which it occurs. The possible mechanism for the formation of superfluidity will be discussed in Part III.

\section{REFERENCES}

[1] Onnes H.K. : Comm.Phys.Lab.,Univ.Leiden, N119, 120,122 (1911)

[2] Ginsburg V.L. : Physics-Uspekhi, 170,N6, 619-630 (2000)

[3] de Nobel, J Phys.Today 49,(9) 40 (1996)

[4] W.Meissner, R.Ochsenfeld, Naturwiss., 21, 787 (1933)

[5] H.London, F.London: Proc.Roy. Soc., A149, 71 (1935)

Physica, 2, 341 (1935)

[6] de Gennes P.G. : Superconductivity of metals and alloys, New York, 787 (1966)

[7] Ketterson J.B. and Song S.N.: Superconductivity, Cambridge (1999)

[8] Linton E.A. : Superconductivity, London: Mathuen and Co.LTDA, NY: John Wiley and Sons Inc., (1964)

[9] Shablo A.A. et al: Letters JETPh, v.19, 7,p.457-461 (1974)

[10] Sharvin D.Iu. and Sharvin Iu.V.: Letters JETPh, v.34, 5, p.285-288 (1981)

[11] Ginsburg V.L., Landau L.D.: JETP, 20, 1064 (1950)

[12] Phillips N.E.: Phys.Rev.B, 114, 676 (1959).

[13] Landau L.D. : JETP, 11, 592 (1941)

[14] Khalatnikov I.M.: Introduction into theory of superfluidity, Moscow, Nauka, (1965)

[15] Feynman R., Statistical Mechanics, Addison Wesley , (1981)

[16] Mineev V.P.: Physics-Uspekhi, 139, p.303,(1983)

[17] Volovik G.E.: Physics-Uspekhi, 143, p.143, (1984)

[18] Likhachev A.G., Polushkin V.N., Uchaikin, Vasiliev B.V.: Magnetocardiometer based on a single-hole high-Tc SQUID, Supercond. Sci. Technol. 3, 148151, (1990) 
[19] Pool Ch.P.Jr : Handbook of Superconductivity, Academic Press, (2000)

[20] Bethe H., Sommerfeld A. : Elektronentheorie der Metalle, Springer, 1933.

[21] Wilson A.H. : Theory of metals, (Cambrige University Press, London, 1938.

[22] Maxwell E. : Phys.Rev.,78,p 477(1950).

[23] Serin et al : Phys.Rev.B,78,p 813(1950). 